\title{
Spatially-averaged flows over mobile rough beds: definitions, averaging theorems, and conservation equations
}

\author{
Vladimir Nikora ${ }^{1}$, Francesco Ballio², Stephen Coleman ${ }^{3}$, Dubravka Pokrajac ${ }^{4}$
}

\begin{abstract}
The paper reports the double-averaged (in space and in time) hydrodynamic equations for mobile-boundary conditions which are derived based on the refined double-averaging theorems, modified Reynolds decomposition, and improved definitions of the spatial and time bed porosities. The obtained double-averaged conservation equations provide a mathematical framework for studying mobile-boundary flows such as gravel-bed rivers during flood events or flows over vegetated beds. These equations will help in designing measurement campaigns for obtaining mobile-bed data and their interpretation and parameterisation, eventually leading to improved and more robust predictive models.
\end{abstract}

CE database subject headings: Open-channel flow; Turbulence; Sediment transport; Vegetated flows

Author keywords: Open-channel flow; Turbulence; Sediment transport; Vegetated flows

\section{Introduction}

Environmental flows such as overland flows, rivers, estuaries or coastal flows can often be classified as low-submergence rough-bed flows with high levels of heterogeneity in time-averaged hydrodynamic fields due to the effects of roughness elements, especially profound in the near-bed region. These flows are typically described by the Reynolds averaged Navier-Stokes equations, which deal with time- (ensemble-) averaged variables and involve no spatial averaging. Such an approach, however, is often inconvenient, to say the least, due to the complex and often mobile boundary conditions that lead to the high flow heterogeneity. The key drawbacks of the RANS-based approaches in relation to rough-bed flows have been discussed at length in Nikora et al. (2007a). It has been argued that to resolve the problem theoretically, time (or ensemble) averaging of the hydrodynamic equations should be supplemented by volume averaging or area averaging in the plane parallel to the mean (smoothed) bed surface. Conceptually, the double-averaged (in both time and space) equations relate to the time-(ensemble)-averaged equations as the time-averaged equations relate to the Navier-Stokes and advection-diffusion equations for instantaneous hydrodynamic variables. The development of this methodology for rough-bed flows was initiated by atmospheric scientists for describing turbulent flows within and above terrestrial canopies such as forests or bushes (Wilson and Shaw 1977; Raupach and Shaw 1982; Finnigan 1985, 2000; Poggi et al. 2004), and later it was adopted in studies of water flows (e.g., Gimenez-Curto and Corniero Lera 1996; Lopez and Garcia 2001; Nikora et al. 2001, 2007a,b; Pokrajac et al. 2008; Nepf 2012).

\footnotetext{
${ }^{1}$ Professor, School of Engineering, University of Aberdeen, UK (corresponding author). Email: v.nikora@abdn.ac.uk

${ }^{2}$ Professor, Department of Civil and Environmental Engineering, Politecnico di Milano, Italy. Email: francesco.ballio@polimi.it

${ }^{3}$ Associate Professor, Department of Civil and Environmental Engineering, University of Auckland, New Zealand. Email: s.Coleman@auckland.ac.nz

${ }^{4}$ Reader, School of Engineering, University of Aberdeen, UK. Email: d.pokrajac@abdn.ac.uk
} 
Applications of the double-averaging approach to a wide range of flows, from porous media flows to rough-bed open-channel flows to atmospheric boundary layers, have recently been discussed in a special issue of Acta Geophysica (Nikora and Rowinski 2008) that highlights the main advantages of this methodology, i.e.: (a) rigor and self-consistency; (b) refined definitions for roughbed flows such as flow uniformity, two-dimensionality, and the bed shear stress; (c) a consistent link between spatially-averaged roughness parameters, bed shear stress, and double-averaged flow variables; (d) explicit accounting for the viscous drag, form drag and form-induced stresses and substance fluxes as a result of rigorous derivation rather than intuitive reasoning; (e) framework for scaling considerations and parameterizations based on double-averaged variables; and (f) the possibility for the rigorous scale partitioning of the roughness parameters and flow properties. These advantages underpin use of the double-averaged hydrodynamic equations in developing numerical models and associated closures for environmental rough-bed flows; designing laboratory, field, and numerical experiments; data analysis and interpretation; and guiding conceptual developments and parameterizations.

The main achievements to date in this research area relate to fixed-bed flows while mobile-bed flows still represent a major challenge in terms of both theoretical frameworks and experimental data. To address this issue, Nikora et al. (2007a) presented the double-averaged hydrodynamic equations and introduced two parameters characterising mobile bed conditions: the space and time bed porosities. Since publication of that paper, the authors have received feedback from colleagues interested in modelling mobile-bed flows that highlights the need for clarification of the doubleaveraging methodology for mobile-bed conditions.

The goal of this paper is therefore to refine the double-averaged hydrodynamic equations for mobile-bed conditions by clarifying key ingredients involved in the derivation (Nikora et al., 2007a): (1) averaging operators and space and time bed porosities; (2) equations that link double-averaged derivatives to derivatives of the double-averaged variables, known as the averaging theorems; and (3) modified Reynolds decomposition of instantaneous variables. The derivation starts with presenting instantaneous variables in the hydrodynamic equations using the modified Reynolds decomposition, followed by applying averaging procedures for each term of the equation, similar to how the Reynolds-averaged equations are obtained. The double-averaging theorems play the role of the Reynolds averaging rules in this derivation. There are a number of options for derivation of the double-averaging theorems (e.g., Raupach and Shaw 1982; Finnigan 1985; Lien et al. 2005; Kono et al. 2010). Nikora et al. (2007a) in their derivation of the double-averaging theorems and doubleaveraged equations have employed an approach suggested by Gray and Lee (1977) for local volume averaging of instantaneous variables of multiphase systems. Nikora et al. (2007a) extended this approach to cover double-averaging, considering both superficial averaging (over the whole averaging domain) and intrinsic averaging (over the sub-domain occupied by fluid only; see next 79 section for more specific definitions). According to Gray and Lee (1977), superficial $\left(\langle\theta\rangle_{s}\right)$ and 80 intrinsic $(\langle\theta\rangle)$ spatial averages of a variable $\theta$ are expressed as:

$\langle\theta\rangle_{s}\left(x_{i}, t\right)=\frac{1}{V_{o}} \int_{V_{o}} \theta\left(x_{i}+\xi_{i}, t\right) \gamma\left(x_{i}+\xi_{i}, t\right) d V=\frac{1}{V_{o}} \int_{V_{f}} \theta\left(x_{i}+\xi_{i}, t\right) d V$

$\langle\theta\rangle\left(x_{i}, t\right)=\frac{1}{V_{f}} \int_{V_{o}} \theta\left(x_{i}+\xi_{i}, t\right) \gamma\left(x_{i}+\xi_{i}, t\right) d V=\frac{1}{V_{f}} \int_{V_{f}} \theta\left(x_{i}+\xi_{i}, t\right) d V$

where $\gamma\left(x_{i}, t\right)=\mathrm{a}$ 'clipping' or 'distribution' function equal to 1 in the fluid and 0 otherwise; $V_{f}=\int_{V_{o}} \gamma\left(x_{i}+\xi_{i}, t\right) d V=$ fluid volume within the total averaging domain of volume $V_{o}$; and angular position $x_{i}$ and a local co-ordinate system $\xi_{i}$ is used for integration (Fig. 1); resulting averaged 
variables are assigned to the centres of the averaging domains. In studies of turbulent spots embedded in non-turbulent environments (e.g., Antonia and Atkinson 1974) and in micromechanics (e.g., Torquato 2002), the function $\gamma\left(x_{i}, t\right)$ is also known as the intermittency function $I\left(x_{i}, t\right)$, while an average of $I\left(x_{i}, t\right)$ is often called the intermittency fraction (e.g., Field and Grigoriu, 2010). Gray and Lee (1977) demonstrated that $\partial \gamma / \partial x_{i}=n_{i} \delta\left(x_{i}-x_{s i}\right)$ and $\partial \gamma / \partial t=-v_{i} \partial \gamma / \partial x_{i}=-v_{i} n_{i} \delta\left(x_{i}-x_{s i}\right)$, where $\delta$ = three-dimensional analogue of the Dirac delta-function, $x_{s i}=$ the coordinates of the flowsolid interface, $n_{i}=$ unit vector normal to the bed surface and directed into fluid, and $v_{i}=$ velocity vector of the fluid-solid interface. Gray and Lee's (1977) relationships were reinforced and further generalised by Kinnmark and Gray (1984). Operators similar to (1a) and (1b) can also be introduced for intrinsic and superficial time-averaging as well as for space-time averaging. These operators will be employed in our derivations below.

We first introduce definitions related to forms of averaging, bed porosities, and their interrelations. These considerations are required to eliminate potential confusion between different forms of averaging and bed porosities. Then, we consider the averaging operators, double-averaging theorems, modified Reynolds decomposition, and hydrodynamic equations for mobile-bed conditions, followed by a brief outline of potential applications of the equations.

\section{Averaging Procedures, Bed Porosities, Intrinsic and Superficial Quantities}

\section{Forms of Averaging}

The forms of averaging used in this paper include:

Superficial averaging: averaging of a variable over the whole domain (spatial, time or both). This averaging form is indicated by the index s; e.g., as in equation (1).

Intrinsic averaging: averaging of a variable over the sub-domain (spatial, time or both) within which this variable is appropriately defined or marked with a special feature (e.g., presence of water within a sub-domain).

Time averaging: averaging over time; defined with an overbar (e.g., superficial $\bar{\theta}^{s}$ and intrinsic $\bar{\theta}$ ).

Spatial averaging: averaging over space; defined with angular brackets (e.g., superficial $\langle\theta\rangle_{s}$ and intrinsic $\langle\theta\rangle$ ).

Space-time averaging: simultaneous averaging over space and time; defined with rectangular brackets (e.g., superficial $[\theta]_{s}$ and intrinsic $[\theta]$ ). This is probably the most robust form of doubleaveraging.

Consecutive time-space averaging: first averaging over time and then over space (e.g., superficial $\left\langle\bar{\theta}^{s}\right\rangle_{s}$ and intrinsic $\langle\bar{\theta}\rangle$ ). This is another form of the double-averaging.

Consecutive space-time averaging: first averaging over space and then over time (e.g., superficial ${\overline{\langle\theta\rangle_{s}}}^{s}$ and intrinsic $\overline{\langle\theta\rangle}$ ). This is an alternative to the consecutive time-space averaging defined above.

The averaging forms defined above are equally applicable to any hydrodynamic variable such as flow velocity, pressure, or substance concentration. The selection of the shape and dimensions of the 
136

137

138

139

140

141

142

143

144

145

146

147

148

149

150

151

152

153

154

155

156

157

158

159

160

161

162

163

164

165

166

167

168

169

170

171

172

173

174

175

176

177

178

179

spatial averaging domain and the averaging time depends on the roughness geometry and the turbulence structure (particularly on their characteristic scales) as well as on the magnitudes and scales of the spatial and temporal gradients of the mean flow. These issues have been discussed in detail in Nikora et al. (2007a). Here we only mention that in most environmental flows there are strong gradients in flow properties in the vertical direction, especially near the bed, and therefore the volume-averaging domain should be designed as a thin slab parallel to the average bed (Fig. 1), being much thinner than the roughness elements. The dimensions of the averaging domain in the plane parallel to the average bed should be much larger than the dominant roughness scales, but much smaller than the large-scale features in bed topography. For example, for gravel-bed rivers it should be much larger than gravel particles, but much smaller than sizes of riffles or pools. The averaging time should well exceed the integral time scales of turbulence, still being much shorter than the duration of hydrological events such as floods. The exact shape and dimensions of the averaging domain and the averaging time may vary depending on the problem under consideration.

Specific mathematical definitions for superficial and intrinsic quantities used in this paper will be given in the following sections. Note that to derive the double-averaged equations, Nikora et al. (2007a) essentially used consecutive time-space averaging that accounts for the long-term tradition in data collection and analysis which have been largely motivated by the RANS methodology. In general, however, the appearance of the averaging theorems and the double-averaged hydrodynamic equations, as well as the physical meaning of the double-averaged quantities, may depend on the averaging approach, i.e., simultaneous space-time averaging; consecutive time-space averaging; or consecutive space-time averaging, as discussed later in the paper.

\section{Forms of 'Bed Porosity' (Roughness Geometry Function)}

The bed porosity or roughness geometry function (as introduced in Nikora et al. 2001) can be defined in a number of ways, depending on the problem under consideration. In relation to double-averaging, the bed porosity appears in the double-averaged hydrodynamic equations as a parameter representing the bed geometry. A selected set of definitions for bed porosities, relevant to our considerations, is introduced below. Fig. 2 provides sketches that illustrate porosities introduced in this and the following sections.

Space-time porosity $\phi_{V T}\left(x_{i}, t\right)$ represents the ratio of the part of the total averaging domain occupied by fluid (i.e., $\left.\int_{T_{o}} \int_{V_{o}} \gamma\left(x_{i}+\xi_{i}, t+\tau\right) d V d t\right)$ to the size of this domain $V_{o} T_{o}$, i.e.:

$\phi_{V T}\left(x_{i}, t\right)=[\gamma]_{s}=\left\langle\bar{\gamma}^{s}\right\rangle_{s}={\overline{\langle\gamma\rangle_{s}}}^{s}=\frac{1}{T_{o}} \frac{1}{V_{o}} \int_{T_{o}} \int_{V_{o}} \gamma\left(x_{i}+\xi_{i}, t+\tau\right) d V d \tau$

where $T_{o}=$ the averaging period; and $V_{o}=$ the size of the spatial averaging domain, i.e., a spatial component of the total averaging domain $V_{o} T_{o}$. Similar to the spatial averaging (see Eq. (1)), the integration time domain of Eq. (2) is centered at position $t$ and a local time co-ordinate $\tau$ is used for integration; resulting time-averaged variables are assigned to the centers of the averaging domains. In equations that follow, we omit $\xi_{i}$ and $\tau$ for brevity. Equation (2) shows that all three superficial forms of double-averaging for $\gamma$ (i.e., space-time, consecutive time-space, and consecutive spacetime) are identical while the intrinsic space-time average is always $[\gamma] \equiv 1$. The meaning of the quantity $\int_{T_{o}} \int_{V_{o}} \gamma\left(x_{i}, t\right) d V d t$ will be clarified at the end of next section. 
180 Local time porosity $\phi_{T}\left(x_{i}, t\right)$ is defined as the ratio of the total period of time $T_{f}=\int_{T_{o}} \gamma\left(x_{i}, t\right) d t$ (not 181 necessarily continuous) when water passes through a fixed point $x_{i}$, to the total duration $T_{o}$ of observation, i.e.:

$\phi_{T}\left(x_{i}, t\right)=\bar{\gamma}^{s}=\frac{1}{T_{o}} \int_{T_{o}} \gamma\left(x_{i}, t\right) d t$

Note that the quantity $\phi_{T}\left(x_{i}, t\right)=\bar{\gamma}^{s}$ involves no spatial averaging. In the case of the fixed rough bed, we have either $\bar{\gamma}^{s}=0$ when a point is embedded in the solid phase, or $\bar{\gamma}^{s}=1$ when the point is in the region filled with water. For mobile beds where a fixed point is intermittently occupied by solids or water, we have $0 \leq \bar{\gamma}^{s} \leq 1$. Note also that $\bar{\gamma} \equiv 1$. As mentioned above, in turbulence studies and micromechanics, an analogue of $\bar{\gamma}^{s}$ is known as the intermittency fraction. Recently, a similar parameter has been successfully used to study intermittency in bed particle motion (Radice and Ballio 2008).

Instantaneous space porosity $\phi_{V}\left(x_{i}, t\right)$ represents the ratio of the fluid volume $V_{f}=\int_{V_{o}} \gamma\left(x_{i}, t\right) d V$ to the total volume of the averaging domain $V_{o}$ at a moment in time $t$, i.e.:

$$
\phi_{V}\left(x_{i}, t\right)=\langle\gamma\rangle_{s}=\frac{1}{V_{o}} \int_{V_{o}} \gamma\left(x_{i}, t\right) d V
$$

Note that the intrinsic spatial average of the clipping function is always 1, i.e., $\langle\gamma\rangle \equiv 1$. In addition to the space-time porosity $\phi_{V T}\left(x_{i}, t\right)$, the local time porosity $\phi_{T}\left(x_{i}, t\right)$, and the instantaneous space (volumetric) porosity $\phi_{V}\left(x_{i}, t\right)$, introduced above, one may also employ a 'plane' porosity $\phi_{A}\left(x_{i}, t\right)$, which is the ratio of the area $A_{f}$ occupied by fluid within a plane averaging domain to its total area $A_{o}$, i.e.:

$$
\phi_{A}\left(x_{i}, t\right)=\langle\gamma\rangle_{A s}=\frac{1}{A_{o}} \int_{A_{0}} \gamma\left(x_{i}, t\right) d A
$$

where $A_{f}=\int_{A_{0}} \gamma\left(x_{i}, t\right) d A=$ area occupied by fluid. In Nikora et al. (2001), the parameter $\phi_{A}\left(x_{i}, t\right)$ was defined as the roughness geometry function $A$ to describe the flow - 'rough' bed interface. As in previous cases, $\langle\gamma\rangle_{A} \equiv 1$.

\section{Some Interrelations between Time, Space, and Space-Time Porosities}

The physical meanings of the porosities introduced in the previous section can be better seen through relationships between them. The space-time porosity $\phi_{V T}\left(x_{i}, t\right)$, introduced by Eq. (2), can be expressed as:

$$
\phi_{V T}=[\gamma]_{s}=\left\langle\bar{\gamma}^{s}\right\rangle_{s}={\overline{\langle\gamma\rangle_{s}}}^{s}=\frac{1}{V_{o}} \frac{1}{T_{o}} \int_{V_{o}} \int_{T_{o}} \gamma\left(x_{i}, t\right) d t d V=\frac{1}{V_{o}} \int_{V_{o}} \bar{\gamma}^{s} d V=\frac{1}{V_{o}} \int_{V_{m}} \bar{\gamma}^{s} d V+\frac{1}{V_{o}} \int_{V_{o}-V_{m}} \bar{\gamma}^{s} d V
$$


where $V_{m}=$ the part of $V_{o}$ that has been 'visited' by fluid, even briefly, within $T_{o} ; \frac{1}{V_{o}} \int_{V_{o}-V_{m}} \bar{\gamma}^{s} d V=0$ as $\bar{\gamma}^{s}=0$ everywhere within $\left(V_{o}-V_{m}\right)$; and $\phi_{V m}=V_{m} / V_{o}$ is the space porosity based on non-zero $\bar{\gamma}^{s}$. The quantity $\left(V_{o}-V_{m}\right)$ is the remaining part of $V_{o}$ that has never been 'visited' by fluid, even briefly, within $T_{o}$, and thus represents the total volume of permanent solid 'islands' within $V_{o}$. For fixed ('frozen') beds, $\left\langle\phi_{T}\right\rangle \equiv 1$ and therefore $\phi_{V T}=\phi_{V m}\left\langle\phi_{T}\right\rangle=\phi_{V m}=\phi_{V}$. For 'spatially homogeneous' mobile beds (i.e., with no permanent solid 'islands' within the averaging domain), $\bar{\gamma}^{s} \neq 0$ everywhere within $V_{o}$, and thus $V_{m}=V_{o}$ and $\phi_{V m}=1$, giving $\phi_{V T}=\phi_{V m}\left\langle\phi_{T}\right\rangle=\left\langle\phi_{T}\right\rangle$. The general case of $\phi_{V T}=\phi_{V m}\left\langle\phi_{T}\right\rangle$ is intermediate between these two extremes.

In addition, we can consider $\phi_{V T}$ in terms of a porosity that reflects potential existence of 'solid islands' in time, i.e.:

$\phi_{V T}=[\gamma]_{s}=\left\langle\bar{\gamma}^{s}\right\rangle_{s}={\overline{\langle\gamma\rangle_{s}}}^{s}=\frac{1}{T_{o}} \frac{1}{V_{o}} \int_{T_{o}} \int_{V_{o}} \gamma\left(x_{i}, t\right) d V d t=\frac{1}{T_{o}} \int_{T_{o}}\langle\gamma\rangle_{s} d t=\frac{1}{T_{o}} \int_{T_{m}}\langle\gamma\rangle_{s} d t+\frac{1}{T_{o}} \int_{T_{o}-T_{m}}\langle\gamma\rangle_{s} d t$

where $T_{m}=$ time period (not necessarily continuous) within the total averaging period $T_{o}$ when a part (even very small) of $V_{o}$ contained fluid; $\frac{1}{T_{o}} \int_{T_{o}-T_{m}}\langle\gamma\rangle_{s} d t=0$ as $\langle\gamma\rangle_{s}=0$ within $\left(T_{o}-T_{m}\right)$; and $\phi_{T m}=T_{m} / T_{o}$ is the time porosity based on non-zero $\langle\gamma\rangle_{s}$. The quantity $\left(T_{o}-T_{m}\right)$ is the remaining part of $T_{o}$ when there is no fluid anywhere within the domain $V_{o}$. For fixed ('frozen') beds, $\phi_{T m} \equiv 1$ and therefore $\phi_{V T}=\phi_{T m} \bar{\phi}_{V}=\bar{\phi}_{V}=\phi_{V}$, as expected. At the other extreme of temporarily homogeneous mobile beds (i.e., with no solid 'islands' in time or, in other words, with no instance of a completely 'solid' domain), $\phi_{T m} \equiv 1$ and therefore $\phi_{V T}=\phi_{T m} \bar{\phi}_{V}=\bar{\phi}_{V}$.

To summarise, in general the space-time porosity $\phi_{V T}$ can be expressed as $\phi_{V T}=\left\langle\bar{\gamma}^{s}\right\rangle_{s}=\phi_{V m}\left\langle\phi_{T}\right\rangle$ and $\phi_{V T}=\overline{\langle\gamma}_{s}^{s}=\phi_{T m} \bar{\phi}_{V}$, giving $\phi_{V m}\left\langle\phi_{T}\right\rangle=\phi_{T m} \bar{\phi}_{V}$. For the special case of homogeneously mobile beds (with no 'solid islands' in time or in space), we have $\phi_{V T}=\left\langle\bar{\gamma}^{s}\right\rangle_{s}=\left\langle\phi_{T}\right\rangle$ and $\phi_{V T}=\overline{\langle\gamma\rangle}_{s}^{s}=\bar{\phi}_{V}$, giving $\phi_{V T}=\left\langle\phi_{T}\right\rangle=\bar{\phi}_{V}$.

With the above definitions, another useful relationship is $\int_{T_{o}} \int_{V_{o}} \gamma\left(x_{i}, t\right) d V d t=\bar{V}_{f} T_{m}=V_{m}\left\langle T_{f}\right\rangle$ that clarifies the meaning of the quantity $\int_{T_{o}} \int_{V_{o}} \gamma\left(x_{i}, t\right) d V d t$ in the definition of $\phi_{V T}$ in Eq. (2). The forms of bed porosity introduced in this subsection will be used below in establishing relations between different forms of averaging as well as in the double-averaging theorems and in the doubleaveraged hydrodynamic equations.

\section{Specific Definitions for Superficial and Intrinsic Averages}


268

269

270

271

272

273

\section{Superficial averaging}

The superficial double-averaged quantity can be defined as a generalisation of $\langle\theta\rangle_{s}$ in Eq. (1), i.e.:

$$
[\theta]_{s}=\left\langle\bar{\theta}^{s}\right\rangle_{s}={\overline{\langle\theta\rangle_{s}}}^{s}=\frac{1}{T_{o}} \frac{1}{V_{o}} \int_{T_{o} V_{o}} \int_{V_{o}} \theta \gamma\left(x_{i}, t\right) d V d t
$$

As can be seen in Eq. (8), all three forms of double-averaging (i.e., space-time, consecutive timespace, and consecutive space-time) are identical for superficial averages. They may not be identical for intrinsic averages, however, as shown below.

\section{Intrinsic space-time averaging}

This form of averaging is defined as:

$[\theta]=\frac{1}{\int_{T_{o}} \int_{V_{o}} \gamma\left(x_{i}, t\right) d V d t} \int_{T_{o} V_{o}} \theta \gamma\left(x_{i}, t\right) d V d t=\frac{1}{\bar{V}_{f} T_{m}} \int_{T_{o}} \int_{V_{o}} \theta \gamma\left(x_{i}, t\right) d V d t=\frac{1}{V_{m}\left\langle T_{f}\right.} \int_{T_{o}} \int_{V_{o}} \theta \gamma\left(x_{i}, t\right) d V d t$

Then the relation between $[\theta]_{s}$ and $[\theta]$ follows:

$$
[\theta]_{S}=\frac{1}{T_{o}} \frac{1}{V_{o}} \int_{T_{o} V_{V_{o}}} \theta \gamma\left(x_{i}, t\right) d V d t=\frac{\iint_{T_{o}} \gamma\left(x_{i}, t\right) d V d t}{T_{o} V_{o}} \frac{1}{\iint_{T_{o}} \gamma\left(x_{V_{o}}, t\right) d V d t} \int_{T_{o}} \int_{V_{o}} \theta \gamma\left(x_{i}, t\right) d V d t=\phi_{V T}[\theta]
$$

\section{Intrinsic consecutive time-space averaging}

By applying first time averaging and then spatial averaging, the quantity $\langle\bar{\theta}\rangle$ can be defined as:

$$
\langle\bar{\theta}\rangle=\frac{1}{V_{m}} \int_{V_{o}} \frac{1}{T_{f}} \int_{T_{o}} \theta \gamma\left(x_{i}, t\right) d t d V=\frac{1}{V_{m}} \int_{V_{o}} \bar{\theta} d V
$$

The relation between $\left\langle\bar{\theta}^{s}\right\rangle_{s}=[\theta]_{s}={\overline{\langle\theta\rangle_{s}}}^{s}$ and $\langle\bar{\theta}\rangle$ is then derived:

$$
\left\langle\bar{\theta}^{s}\right\rangle_{s}=[\theta]_{s}={\overline{\langle\theta\rangle_{s}}}^{s}=\frac{1}{T_{o}} \frac{1}{V_{o}} \int_{T_{o}} \int_{V_{o}} \theta \gamma\left(x_{i}, t\right) d V d t=\frac{1}{V_{o}} \int_{V_{o}} \frac{1}{T_{o}} \int_{T_{o}} \theta \gamma\left(x_{i}, t\right) d t d V
$$

$$
=\frac{V_{m}}{V_{o}} \frac{1}{V_{m}} \int_{V_{o}} \frac{T_{f}}{T_{o}} \frac{1}{T_{f}} \int_{T_{o}} \theta \gamma\left(x_{i}, t\right) d t d V=\frac{V_{m}}{V_{o}} \frac{1}{V_{m}} \int_{V_{o}} \frac{T_{f}}{T_{o}} \bar{\theta} d V=\phi_{V m}\left\langle\phi_{T} \bar{\theta}\right\rangle
$$

\section{Intrinsic consecutive space-time averaging}

We can define $\overline{\langle\theta\rangle}$ similar to Eq. (11) but with the reverse averaging order, i.e.: 
$\overline{\langle\theta\rangle}=\frac{1}{T_{m}} \int_{T_{o}} \frac{1}{V_{f}} \int_{V_{o}} \theta \gamma\left(x_{i}, t\right) d V d t=\frac{1}{T_{m}} \int_{T_{o}}\langle\theta\rangle d t$

The relation between ${\overline{\langle\theta\rangle_{s}}}^{s}=\left\langle\bar{\theta}^{s}\right\rangle_{s}=[\theta]_{s}$ and $\overline{\langle\theta\rangle}$ can be obtained as:

293

${\overline{\langle\theta\rangle_{s}}}^{s}=\left\langle\bar{\theta}^{s}\right\rangle_{s}=[\theta]_{s}=\frac{1}{T_{o}} \frac{1}{V_{o}} \int_{T_{o} V_{o}} \int_{V} \theta \gamma\left(x_{i}, t\right) d V d t=\frac{1}{T_{o}} \int_{T_{o}} \frac{1}{V_{o}} \int_{V_{o}} \theta \gamma\left(x_{i}, t\right) d V d t$

295

296

$=\frac{T_{m}}{T_{o}} \frac{1}{T_{m}} \int_{T_{o}} \frac{V_{f}}{V_{o}} \frac{1}{V_{f}} \int_{V_{o}} \theta \gamma\left(x_{i}, t\right) d V d t=\frac{T_{m}}{T_{o}} \frac{1}{T_{m}} \int_{T_{o}} \frac{V_{f}}{V_{o}}\langle\theta\rangle d t=\phi_{T m} \overline{\phi_{V}\langle\theta\rangle}$

To summarise, from the above relationships it follows that:

$[\theta]_{s}=\left\langle\bar{\theta}^{s}\right\rangle_{s}={\overline{\langle\theta\rangle_{s}}}^{s}=\phi_{V T}[\theta]=\phi_{V m}\left\langle\phi_{T}\right\rangle[\theta]=\phi_{T m} \bar{\phi}_{V}[\theta]=\phi_{V m}\left\langle\phi_{T} \bar{\theta}\right\rangle=\phi_{T m} \overline{\phi_{V}\langle\theta\rangle}$

where we use the equality $\phi_{V T}=\phi_{V m}\left\langle\phi_{T}\right\rangle=\phi_{T m} \bar{\phi}_{V}$ derived in Eqs. (6) and (7). Equations (15) show that the three different forms of intrinsic averages relate to each other as $\phi_{V m}\left\langle\phi_{T}\right\rangle[\theta]=\phi_{T m} \bar{\phi}_{V}[\theta]=\phi_{V m}\left\langle\phi_{T} \bar{\theta}\right\rangle=\phi_{T m} \overline{\phi_{V}\langle\theta\rangle}$, i.e., in general, they are not identical. For spatially non-correlated $\phi_{T}$ and $\bar{\theta}$, and for time non-correlated $\phi_{V}$ and $\langle\theta\rangle$, equations (15) simplify to:

$$
\phi_{V m}\left\langle\phi_{T}\right\rangle[\theta]=\phi_{T m} \bar{\phi}_{V}[\theta]=\phi_{V m}\left\langle\phi_{T}\right\rangle\langle\bar{\theta}\rangle=\phi_{T m} \overline{\phi_{V}} \overline{\langle\theta\rangle}
$$

from which we can conclude that for the uncorrelated pairs ( $\phi_{T}$ and $\bar{\theta}$ ) and ( $\phi_{V}$ and $\langle\theta\rangle$ ) the three forms of intrinsic averages are identical, i.e., $[\theta]=\langle\bar{\theta}\rangle=\overline{\langle\theta\rangle}$. The same applies, of course, for the fixed bed conditions, for which $\left\langle\phi_{T}\right\rangle \equiv 1, \phi_{V m}=\phi_{V}, \phi_{T m} \equiv 1, \overline{\phi_{V}}=\phi_{V}$, and thus $[\theta]=\langle\bar{\theta}\rangle=\overline{\langle\theta\rangle}$ again.

\section{Double-Averaging Theorems}

\section{Double-Averaging Theorems for Superficial Quantities}

We first review the derivation of the double-averaging theorems for the superficial variables, refining Nikora et al.'s (2007a) approach. Using Eq. (8) and Gray and Lee's (1977) relationships as outlined in the Introduction, we can obtain the averaging theorem for the time derivative as:

$$
\left[\frac{\partial \theta}{\partial t}\right]_{s}=\left\langle\frac{\overline{\partial \theta}^{s}}{\partial t}\right\rangle_{s}=\overline{\left\langle\frac{\partial \theta}{\partial t}\right\rangle_{s}^{s}}=\frac{1}{T_{o}} \frac{1}{V_{o}} \int_{T_{o}} \int_{V_{o}} \frac{\partial \theta}{\partial t} \gamma\left(x_{i}, t\right) d V d t=\frac{1}{T_{o}} \frac{1}{V_{o}} \int_{T_{o}} \int_{V_{o}} \frac{\partial \theta \gamma}{\partial t} d V d t-\frac{1}{T_{o}} \frac{1}{V_{o}} \int_{T_{o}} \int_{V_{o}} \theta \frac{\partial \gamma}{\partial t} d V d t
$$

$$
=\frac{\partial}{\partial t}\left(\frac{1}{T_{o}} \frac{1}{V_{o}} \int_{T_{o}} \int_{V_{o}} \theta \gamma d V d t\right)+\frac{1}{T_{o}} \frac{1}{V_{o}} \int_{T_{o}}\left(\int_{V_{o}} \theta v_{i} n_{i} \delta\left(x_{i}-x_{s i}\right) d V\right) d t
$$

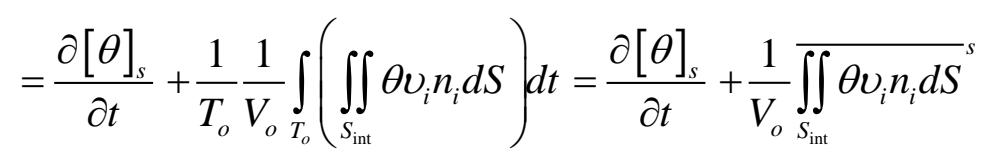


For the spatial derivative, it follows, similarly, that:

$\left[\frac{\partial \theta}{\partial x_{i}}\right]_{s}=\left\langle\frac{\overline{\partial \theta}^{s}}{\partial x_{i}}\right\rangle_{s}=\overline{\left\langle\frac{\partial \theta}{\partial x_{i}}\right\rangle_{s}^{s}}=\frac{1}{T_{o}} \frac{1}{V_{o}} \int_{T_{o}} \int_{V_{o}} \frac{\partial \theta}{\partial x_{i}} \gamma\left(x_{i}, t\right) d V d t=\frac{1}{T_{o}} \frac{1}{V_{o}} \int_{T_{o} V_{o}} \frac{\partial \theta \gamma}{\partial x_{i}} d V d t-\frac{1}{T_{o}} \frac{1}{V_{o}} \int_{T_{o}} \int_{V_{o}} \theta \frac{\partial \gamma}{\partial x_{i}} d V d t$

$=\frac{\partial}{\partial x_{i}}\left(\frac{1}{T_{o}} \frac{1}{V_{o}} \int_{T_{o} V_{o}} \theta \gamma d V d t\right)-\frac{1}{T_{o}} \frac{1}{V_{o}} \int_{T_{o}}\left(\int_{V_{o}} \theta n_{i} \delta\left(x_{i}-x_{s i}\right) d V\right) d t$

$=\frac{\partial[\theta]_{S}}{\partial x_{i}}-\frac{1}{T_{o}} \frac{1}{V_{o}} \iint_{T_{o}}\left(\iint_{S_{\mathrm{int}}} \theta n_{i} d S\right) d t=\frac{\partial[\theta]_{S}}{\partial x_{i}}-\frac{1}{V_{o}} \bar{\int}_{S_{\mathrm{int}}} \theta n_{i} d S$

Thus, we arrive at the following general double-averaging theorems for superficial derivatives:

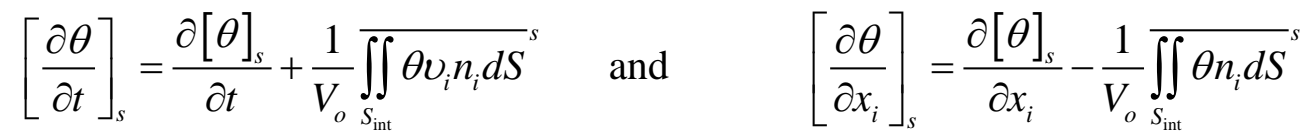

which are suitable for both fixed-bed and mobile-bed conditions. Note that although Nikora et al. (2007a) used consecutive time-space averaging, equations (19) are identical to equations (6a) in Nikora et al. (2007a), as a consequence of $[\theta]_{s}=\left\langle\bar{\theta}^{s}\right\rangle_{s}={\overline{\langle\theta\rangle_{s}}}^{s}$.

\section{Double-Averaging Theorems for Intrinsic Quantities}

Using the relation $[\theta]_{S}=\phi_{V T}[\theta]$ from Eq. (10), we can obtain from (19) the general doubleaveraging theorems for intrinsic derivatives as:

$\left[\frac{\partial \theta}{\partial t}\right]=\frac{1}{\phi_{V T}} \frac{\partial \phi_{V T}[\theta]}{\partial t}+\frac{1}{\phi_{V T} V_{o}}{\iint_{S_{\text {int }}} \theta v_{i} n_{i} d S}_{s}^{s}$ and $\left[\frac{\partial \theta}{\partial x_{i}}\right]=\frac{1}{\phi_{V T}} \frac{\partial \phi_{V T}[\theta]}{\partial x_{i}}-\frac{1}{\phi_{V T} V_{o}} \int_{S_{\text {int }}} \theta n_{i} d S$

Taking into account that $\phi_{V T} / \phi_{V m}=\left\langle\phi_{T}\right\rangle$ and $[\theta]=\left\langle\phi_{T} \bar{\theta}\right\rangle /\left\langle\phi_{T}\right\rangle$ that follow from equations (6) and (15), one can also obtain from (20):

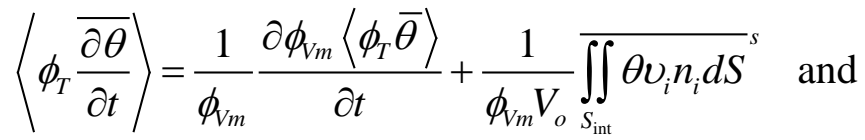

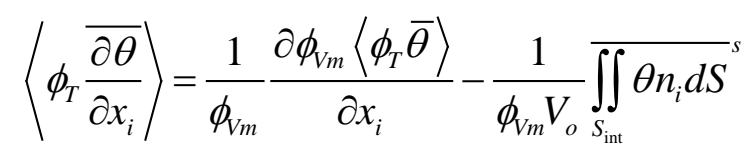

Equations (21) are consistent with equations (6b) in Nikora et al. (2007a) that were derived using, implicitly, consecutive time-space averaging. Although being algebraically identical to those in Nikora et al. (2007a, Eq 6b), equations (21) now involve spatial and time bed porosities that have been explicitly derived in this paper for mobile-bed conditions. Thus, equations (20) and (21) update those given in Nikora et al. (2007a) by refining the meanings of variables and parameters involved in the averaging procedures, thereby making their use and parameterisations for mobile-bed conditions clarified. It should be highlighted that the spatial averaging theorems of (19) and (20), as well as 
those that follow from them such as (21), are equally applicable for fixed- and mobile-bed conditions.

\section{Modified Reynolds Decomposition}

As outlined in the Introduction, derivation of the double-averaged hydrodynamic equations involves three components, two of which (the averaging operators and theorems) have been revisited in the previous sections. The remaining component, modified Reynolds decomposition of instantaneous variables, can be defined for the discussed forms of double averaging as:

$\theta=[\theta]+\ddot{\theta}=\left\langle\phi_{T} \bar{\theta}\right\rangle /\left\langle\phi_{T}\right\rangle+\ddot{\theta} \quad$ or

$\theta=[\theta]+\ddot{\theta}=\overline{\phi_{V}\langle\theta\rangle} / \bar{\phi}_{V}+\ddot{\theta}$

$\theta=\langle\bar{\theta}\rangle+\tilde{\bar{\theta}}+\theta^{\prime}=\bar{\theta}+\theta^{\prime}$

$\theta=\overline{\langle\theta\rangle}+\langle\theta\rangle^{\prime}+\tilde{\theta}=\langle\theta\rangle+\tilde{\theta}$ for space-time averaging

\section{for consecutive time-space averaging}

for consecutive space-time averaging

where $\ddot{\theta}=$ the deviation of the instantaneous variable $\theta$ from its double-averaged value $[\theta], \tilde{\theta}=$ the deviation of the instantaneous variable $\theta$ from its spatially-averaged instantaneous value $\langle\theta\rangle, \tilde{\bar{\theta}}$ $=$ the deviation of the time-averaged variable $\bar{\theta}$ from its spatially-averaged value $\langle\bar{\theta}\rangle$, and prime indicates deviation from a time-averaged value. Note that to improve consistency in symbols for different forms of averaging we use here $\tilde{\bar{\theta}}$ and $\tilde{\theta}$ instead of $\tilde{\theta}$ and $\hat{\theta}$ employed in Nikora et al. (2007a,b), respectively. For fixed beds or in the case of the time and space porosities being uncorrelated with hydrodynamic variables for mobile beds, the decomposition $\theta=[\theta]+\ddot{\theta}$ merges with the decompositions $\theta=\langle\bar{\theta}\rangle+\tilde{\bar{\theta}}+\theta^{\prime}$ and $\theta=\overline{\langle\theta\rangle}+\langle\theta\rangle^{\prime}+\tilde{\theta}$ (since $[\theta]=\langle\bar{\theta}\rangle=\overline{\langle\theta\rangle}$ ), which can be linked through the double-decomposition of Pedras and de Lemos (2000), as discussed in Nikora et al. (2007a) and explored in Pokrajac et al. (2008), i.e.:

$$
\theta=\langle\bar{\theta}\rangle+\ddot{\theta}=\langle\bar{\theta}\rangle+\tilde{\bar{\theta}}+\theta^{\prime}, \quad \theta=\overline{\langle\theta\rangle}+\ddot{\theta}=\overline{\langle\theta\rangle}+\langle\theta\rangle^{\prime}+\tilde{\theta} \quad \Rightarrow \quad\langle\theta\rangle^{\prime}+\tilde{\theta}=\tilde{\bar{\theta}}+\theta^{\prime}=\ddot{\theta}
$$

\section{Double-Averaged Hydrodynamic Equations}

For the general case of mobile-bed flows, the appearance of the double-averaged equations will depend on an averaging form and associated decomposition of flow variables. For consistency with RANS, and to take advantage of already-available data (which in most cases were collected within a RANS framework), the consecutive time-space form of double averaging and the associated decomposition $\theta=\langle\bar{\theta}\rangle+\tilde{\bar{\theta}}+\theta^{\prime}$ are adopted in the following discussion. In the derivation of the equations below, it is assumed that $\overline{\bar{\theta}}=\bar{\theta},\langle\langle\theta\rangle\rangle=\langle\theta\rangle, \overline{\bar{\theta}}=\tilde{\bar{\theta}},\langle\tilde{\theta}\rangle=0$, and $\overline{\theta^{\prime}}=0$, similar to the Reynolds averaging rules. Thus, using equation (12), i.e., $[\theta]_{s}=\left\langle\bar{\theta}^{s}\right\rangle_{s}=\phi_{V m}\left\langle\phi_{T} \bar{\theta}\right\rangle$, the decomposition $\theta=\langle\bar{\theta}\rangle+\tilde{\bar{\theta}}+\theta^{\prime}$, and the spatial averaging theorems (21), the following doubleaveraged equations can be derived from their counterparts for instantaneous variables as shown below. 
402

403

404

405

406

407

408

409

$410 \quad \frac{\partial \phi_{V m}\left\langle\phi_{T}\right\rangle}{\partial t}+\frac{\partial \phi_{V m}\left\langle\phi_{T} \bar{u}_{i}\right\rangle}{\partial x_{i}}=0$

411

412

413

414

415

416

417

418

419

429

422

423

424

425

426

427

428

$$
\frac{\partial u_{i}}{\partial t}+\frac{\partial u_{i} u_{j}}{\partial x_{j}}=g_{i}-\frac{1}{\rho} \frac{\partial p}{\partial x_{i}}+\frac{\partial}{\partial x_{j}}\left(v \frac{\partial u_{i}}{\partial x_{j}}\right)
$$

the following double-averaged momentum equation is obtained:

$$
\underbrace{\frac{\partial \phi_{V m}\left\langle\phi_{T} \bar{u}_{i}\right\rangle}{\partial t}}_{1}+\underbrace{\frac{\partial \phi_{V m}\left\langle\phi_{T}\right\rangle\left\langle\bar{u}_{i}\right\rangle\left\langle\bar{u}_{j}\right\rangle}{\partial x_{j}}}_{2}=\underbrace{\phi_{V m}\left\langle\phi_{T} g_{i}\right\rangle}_{3}-\underbrace{\frac{1}{\rho} \frac{\partial \phi_{V m}\left\langle\phi_{T} \bar{p}\right\rangle}{\partial x_{i}}}_{4}-\underbrace{\frac{\partial \phi_{V m}\left\langle\phi_{T} \overline{u_{i}^{\prime} u_{j}^{\prime}}\right\rangle}{\partial x_{j}}}_{5}-\underbrace{\frac{\partial \phi_{V m}\left\langle\phi_{T} \tilde{\bar{u}}_{i} \tilde{\bar{u}}_{j}\right\rangle}{\partial x_{j}}}_{6}
$$

$\underbrace{\frac{\partial}{\partial x_{j}}\left(\phi_{V m}\left\langle\phi_{T} v \frac{\overline{\partial u_{i}}}{\partial x_{j}}\right\rangle\right)}_{7}-\underbrace{\frac{\partial \phi_{V m}\left\langle\phi_{T} \tilde{\bar{u}_{i}}\right\rangle\left\langle\bar{u}_{j}\right\rangle}{\partial x_{j}}}_{8}-\underbrace{\frac{\partial \phi_{V m}\left\langle\phi_{T} \tilde{\bar{u}}_{j}\right\rangle\left\langle\bar{u}_{i}\right\rangle}{\partial x_{j}}}_{9}$

Starting with:

$\frac{\partial \rho}{\partial t}+\frac{\partial \rho u_{i}}{\partial x_{i}}=0$

where we use $\phi_{V T}=\phi$ for brevity.

\section{Double-averaged momentum equation}

Using the Navier-Stokes equation as a starting point, i.e.:

$$
+\underbrace{\frac{1}{\rho} \frac{1}{V_{o}}{\overline{\int S_{\text {int }}}}_{\int n_{i} d S}^{s}}_{10}-\underbrace{\frac{1}{V_{o}} \overline{\iint_{S_{\text {int }}}\left(v \frac{\partial u_{i}}{\partial x_{j}}\right) n_{j} d S}}_{11}
$$

Eq. (23b) is derived by applying an operation of superficial double-averaging to each term of the initial momentum equation (23a), and then transforming these terms using the double-averaging theorems (Eqs. 21) supplemented with the decomposition of the instantaneous velocities as $u_{i}=\left\langle\bar{u}_{i}\right\rangle+\tilde{\bar{u}}_{i}+u_{i}^{\prime}$. Terms 1 and 2 in equation (23b) represent local and convective accelerations, 
435

436

437

438

439

440

441

442

443

444

445

446

447

448

448

451

452

453

454

455

456

respectively. The third term is the gravity term; the fourth term is the pressure gradient; the fifth, sixth and seventh terms are contributions from turbulent $\left(\left\langle\phi_{T} \overline{u_{i}^{\prime} u_{j}^{\prime}}\right\rangle\right)$, form-induced $\left(\left\langle\phi_{T} \tilde{\bar{u}}_{i} \tilde{\bar{u}}_{j}\right\rangle\right)$, and viscous fluid stresses, respectively; the eighth and ninth terms represent momentum fluxes (stresses) due to potential spatial correlations between the local time porosity and time-averaged velocities; and the final two terms, i.e., tenth and eleventh, are pressure and viscous drag terms. For the case when spatial correlations between the local time porosity and time-averaged flow parameters can be neglected, equation (23b) can be simplified, using the continuity equation (22c) and the space-time porosity $\phi=\phi_{V T}=\phi_{V m}\left\langle\phi_{T}\right\rangle$, as:

$$
\begin{array}{r}
\frac{\partial\left\langle\bar{u}_{i}\right\rangle}{\partial t}+\left\langle\bar{u}_{j}\right\rangle \frac{\partial\left\langle\bar{u}_{i}\right\rangle}{\partial x_{j}}=g_{i}-\frac{1}{\rho} \frac{1}{\phi} \frac{\partial \phi\langle\bar{p}\rangle}{\partial x_{i}}-\frac{1}{\phi} \frac{\partial \phi\left\langle\overline{u_{i}^{\prime} u_{j}^{\prime}}\right\rangle}{\partial x_{j}}-\frac{1}{\phi} \frac{\partial \phi\left\langle\tilde{\bar{u}}_{i} \tilde{\bar{u}}_{j}\right\rangle}{\partial x_{j}}+\frac{1}{\phi} \frac{\partial}{\partial x_{j}}\left(\phi\left\langle v \frac{\partial \overline{u_{i}}}{\partial x_{j}}\right\rangle\right) \\
\left.+\frac{1}{\rho} \frac{1}{\phi} \frac{1}{V_{o}} \int_{S_{\mathrm{int}}} \int_{p n_{i} d S}^{s}-\frac{1}{\phi} \frac{1}{V_{o}} \int_{S_{\text {int }}} \int v \frac{\partial u_{i}}{\partial x_{j}}\right) n_{j} d S
\end{array}
$$

\section{Double-averaged advection-diffusion equation}

Using the advection-diffusion equation for instantaneous variables as a starting point, i.e.:

$$
\frac{\partial C}{\partial t}+\frac{\partial C u_{j}}{\partial x_{j}}=\frac{\partial}{\partial x_{j}}\left(\chi_{m} \frac{\partial C}{\partial x_{j}}\right)+F
$$

the following double-averaged advection-diffusion equation can be similarly derived:

$$
\begin{aligned}
& \underbrace{\frac{\partial \phi_{V m}\left\langle\phi_{T} \bar{C}\right\rangle}{\partial t}}_{1}+\underbrace{\frac{\partial \phi_{V m}\left\langle\phi_{T}\right\rangle\langle\bar{C}\rangle\left\langle\bar{u}_{j}\right\rangle}{\partial x_{j}}}_{2}=\underbrace{\frac{\partial}{\partial x_{j}}\left(\phi_{V m}\left\langle\phi_{T} \chi_{m} \frac{\overline{\partial C}}{\partial x_{j}}\right\rangle\right)}_{3}-\underbrace{\frac{\partial \phi_{V m}\left\langle\phi_{T} \overline{C^{\prime} u_{j}^{\prime}}\right\rangle}{\partial x_{j}}}_{4}-\underbrace{\frac{\partial \phi_{V m}\left\langle\phi_{T} \tilde{\bar{C}}_{\overline{u_{j}}}\right\rangle}{\partial x_{j}}}_{5} \\
& -\underbrace{\frac{\partial \phi_{V m}\left\langle\phi_{T} \tilde{\bar{C}}\right\rangle\left\langle\bar{u}_{j}\right\rangle}{\partial x_{j}}}_{6}-\underbrace{\frac{\partial \phi_{V m}\left\langle\phi_{T} \tilde{\bar{u}}_{j}\right\rangle\langle\bar{C}\rangle}{\partial x_{j}}}_{7}-\underbrace{\frac{1}{V_{o}} \bar{\int} \int_{S_{\text {int }}}\left(\chi_{m} \frac{\partial C}{\partial x_{j}}\right) n_{j} d S}_{8}+\underbrace{\phi_{V m}\left\langle\phi_{T} \bar{F}\right\rangle}_{9}
\end{aligned}
$$

where $C=$ passive substance concentration; $\chi_{m}=$ molecular diffusion coefficient; and $F=$ source/sink of substance $C$. Terms 1 and 2 in equation (24b) represent local change of concentration and convective transport. The third, fourth, and fifth terms are due to the molecular diffusion, turbulent transport $\left(\left\langle\phi_{T} \overline{C^{\prime} u_{j}^{\prime}}\right\rangle\right)$, and form-induced transport $\left(\left\langle\phi_{T} \tilde{\bar{C}}_{\bar{u}}\right\rangle\right)$, respectively; the sixth and seventh terms represent substance fluxes due to potential spatial correlations between the local time porosity and time-averaged velocities and concentrations; the final two terms, i.e., eighth and ninth, are an interfacial flux term (i.e., heterogeneous reaction rate) and a homogeneous reaction rate, respectively. For the case when spatial correlations between the local time porosity and timeaveraged flow parameters can be neglected, equation (24b) can be simplified, using the continuity equation (22c) and the space-time porosity $\phi=\phi_{V T}=\phi_{V m}\left\langle\phi_{T}\right\rangle$, as: 


$$
\begin{array}{r}
\frac{\partial\langle\bar{C}\rangle}{\partial t}+\left\langle\bar{u}_{j}\right\rangle \frac{\partial\langle\bar{C}\rangle}{\partial x_{j}}=\frac{1}{\phi} \frac{\partial}{\partial x_{j}}\left(\phi\left\langle\chi_{m} \frac{\overline{\partial C}}{\partial x_{j}}\right\rangle\right)-\frac{1}{\phi} \frac{\partial \phi\left\langle\overline{C^{\prime} u_{j}^{\prime}}\right\rangle}{\partial x_{j}}-\frac{1}{\phi} \frac{\partial \phi\left\langle\tilde{\bar{C}}_{\tilde{u}_{j}}\right\rangle}{\partial x_{j}} \\
-\frac{1}{\phi} \frac{1}{V_{o}} \int_{S_{\text {int }}}^{\int}\left(\chi_{m} \frac{\partial C}{\partial x_{j}}\right) n_{j} d S+\langle\bar{F}\rangle
\end{array}
$$

Advection-diffusion equations similar to (24a)-(24c) can also be derived for fine suspended sediments at low concentrations at which the advection-diffusion approximation is appropriate.

Compared to the conventional RANS equations, Eqs. (22)-(24) contain some additional terms such as dispersive or form-induced stresses $\left\langle\phi_{T} \tilde{\bar{u}}_{i} \tilde{\bar{u}}_{j}\right\rangle$ and fluxes $\left\langle\phi_{T} \tilde{\bar{C}} \tilde{\bar{u}}_{j}\right\rangle$ due to spatial correlations of the respective time-averaged velocities and concentration fields; momentum and substance fluxes due to potential spatial correlations between the local time porosity and time-averaged velocities and concentrations; the form drag per unit fluid mass $f_{p_{i}}=-\left(1 / \rho V_{o}\right){\overline{\iint_{S_{\text {int }}} p n_{i} d S}}^{s}$; the viscous drag per unit fluid mass $f_{v i}=\left(1 / V_{o}\right){\overline{\iint_{S_{\text {int }}}\left(v \partial u_{i} / \partial x_{j}\right) n_{j} d S}}^{s}$; and the diffusive flux at the water - bed surface interface $J=\left(1 / V_{o}\right){\overline{\iint_{S_{\text {int }}}\left(\chi_{m} \partial C / \partial x_{j}\right) n_{j} d S}}^{s}$ (including biological surfaces when relevant). The quantities $\left\langle\phi_{T} \tilde{\bar{u}}_{i} \tilde{\bar{u}}_{j}\right\rangle$ and $\left\langle\phi_{T} \tilde{\bar{C}}_{\overline{\bar{u}}}\right\rangle$ in equations (23) and (24) follow from double-averaging, similar to $\overline{u_{i}^{\prime} u_{j}^{\prime}}$ and $\overline{u_{j}^{\prime} C^{\prime}}$ in the time-averaged equations that appear due to time averaging of the Navier-Stokes and advection-diffusion equations for instantaneous variables. Some details on these unconventional terms for fixed-bed flows can be found in Nikora et al. (2007a,b) and Nikora and Rowinski (2008).

When required, the double-averaged hydrodynamic equations can also be formulated within space-time and consecutive space-time averaging frameworks, which are analytically linked to equations (22) to (24) obtained based on consecutive time-space averaging. The appearance of the double-averaged equations for the fixed-bed conditions are equivalent to (22c), (23c), and (24c) where $\phi=\phi_{V T}=\phi_{V m}\left\langle\phi_{T}\right\rangle=\phi_{V}$, as $\left\langle\phi_{T}\right\rangle \equiv 1$ and $\phi_{V m}=\phi_{V}$.

\section{Discussion}

Equations (22c), (23c), and (24c) are identical in appearance to the corresponding equations presented in Nikora et al. (2007a). However, their justification and use for data analysis, interpretation, and modelling of the mobile-bed flows should now be clearer as the meanings of the time and spatial porosities and the potential roles of spatial correlations between the local time porosity and time-averaged velocities and concentrations are now unambiguously defined and explained. It should also be highlighted that equations (22c), (23c), and (24c) are simplified versions of the more general equations (22b), (23b), and (24b) that include the potential effects of spatial correlations between the local time porosity and time-averaged velocities and concentrations (they are neglected in Eqs. (22c), (23c), and (24c)).

The correlations $\left\langle\phi_{T} \tilde{\bar{u}}_{i}\right\rangle$ and $\left\langle\phi_{T} \tilde{\bar{C}}\right\rangle$ in Eqs. (22) to (24) are introduced in this paper for the first time and thus information about them and their gradients is not yet available. These terms have to be quantitatively assessed as there may be situations where they cannot be neglected and thus full equations (22b), (23b), and (24b) have to be employed. Indeed, channel beds of most natural rivers are characterised by roughness patchiness generated by a variety of mechanisms. Examples include particle clusters in gravel-bed rivers (e.g., Papanicolaou et al. 2011), ripple/dune patches in sand-bed rivers (e.g., Aberle et al. 2010), and vegetation patches in low-order rivers (e.g., Nepf 2012). These 
roughness patches (or clusters) introduce some spatial heterogeneity in flow velocity and concentration fields as well as certain spatial variability in bedload and/or vegetation waviness that define the local time porosity $\phi_{T}$. Thus, $\tilde{\bar{u}}_{i}$ and/or $\tilde{\bar{C}}$ can well be spatially correlated with $\phi_{T}$. For instance, considering velocity change within and around a roughness patch on a gravel bed, it is likely that flow velocity above/within the patch is lower than outside it. However, the local time porosity $\phi_{T}$ can be expected to be lower away from a patch centre where flow velocity and thus bedload intensity are enhanced. As a result, the spatial correlation $\left\langle\phi_{T} \tilde{\bar{u}}_{1}\right\rangle$ within an averaging window that includes a patch of increased roughness should be non-zero and negative. Vegetation patches may exhibit an opposite effect as both velocity and time porosity are likely to be minimised within the patch, leading to the positive correlation moment $\left\langle\phi_{T} \tilde{\bar{u}}_{1}\right\rangle$. As river beds often exhibit largescale heterogeneity (e.g., bars, meanders), the spatial gradients of $\left\langle\phi_{T} \tilde{\bar{u}}_{i}\right\rangle$ and $\left\langle\phi_{T} \tilde{\bar{C}}\right\rangle$ can be predicted to be non-zero too. These qualitative speculations require, however, proper quantitative assessments utilising reliable data sets from numerical simulations, laboratory experiments, and field measurements. Until very recently such data sets have been unavailable. However, latest advancements in instrumentation and computational techniques make such assessments in the nearest future realistic. The estimates of $\left\langle\phi_{T} \tilde{\bar{u}}_{i}\right\rangle$ and $\left\langle\phi_{T} \tilde{\bar{C}}\right\rangle$ and their spatial gradients for a range of conditions will provide a base for developing physically-driven parameterisations suitable for applied hydraulic models.

\section{Conclusions}

Double-averaged conservation equations (22) to (24) provide a mathematical framework for studying turbulent mobile-bed flows such as gravel-bed rivers during flood events or flows over vegetated beds. The data on such flows, especially within moving roughness elements, are currently very limited due to both measurement difficulties and the remaining uncertainty of what exactly to measure, interpret, and model. The measurement techniques (e.g., refractive index matching Particle Image Velocimetry or those based on Magnetic Resonance Imaging) and modelling capabilities (e.g., Large Eddy Simulation method) have been improved in recent years and it is likely that extensive data on hydrodynamic variables within mobile roughness elements will appear very soon. Equations (22) to (24) will help in designing measurement and simulation campaigns for obtaining such data and for their interpretation and parameterisation, eventually leading to improved and more robust predictive models.

\section{Acknowledgements}

The work was partly supported by the EPSRC, UK (EP/G056404/1), within the project 'Highresolution numerical and experimental studies of turbulence-induced sediment erosion and near-bed transport', and was also stimulated by the Scientific Research Network WO.027p11N 'The functioning of river ecosystems through plant-flow-soil interactions.' The authors are grateful to the Editor and the reviewers for helpful suggestions that improved the final version of the paper.

\section{References}

Aberle, J., Nikora, V., Henning, M., Ettmer, B, and Hentschel, B. (2010). “Statistical characterization of bed roughness due to bed forms: A field study in the Elbe River at Aken, Germany.” Water Resour. Res., 46, W03521, doi:10.1029/2008WR007406.

Antonia, R.A., and Atkinson, J.D. (1974). "Use of a pseudo-turbulent signal to calibrate an intermittency measuring circuit.” J. Fluid Mech., 64(4), 679-699.

Field Jr., R.V., and Grigoriu, M. (2010). "A Poisson random field model for intermittent phenomena with application to laminar-turbulent transition and material microstructure.” Appl. Math. Modell., doi:10.1016/j.apm.2010.07.059. 
Finnigan, J.J. (1985). “Turbulent transport in flexible plant canopies.” In: The forest-atmosphere interactions, B.A. Hutchinson and B.B. Hicks, eds., D. Reidel Publishing Company, 443-480.

Finnigan, J.J. (2000). “Turbulence in plant canopies.” Annu. Rev. Fluid. Mech., 32, 519-571.

Gimenez-Curto, L.A., and Corniero Lera, M.A. (1996). "Oscillating turbulent flow over very rough surfaces.” J. Geophys. Res., 101(C9), 20,745-20,758.

Gray, W.G., and Lee, P.C.Y. (1977). "On the theorems for local volume averaging of multiphase systems.” Int. J. Multiphase Flow, 3, 333-340.

Kinnmark, I.P.E., and Gray, W.G. (1984). "An exposition of the distribution function used in proving the averaging theorems for multiphase flows.” Adv. Water Resources, 7, 113-115.

Kono,T., Ashie, Y., and Tamura, T. (2010). "Mathematical derivation of spatially-averaged momentum equations for an urban canopy model using underlying concepts of the immersed boundary method.” Boundary-Layer Meteorol., 135, 185-207.

Lien, F.-S., Yee E., and Wilson, J.D. (2005). "Numerical modelling of the turbulent flow developing within and over a 3-d building array, part II: a mathematical foundation for a distributed drag force approach.” Boundary-Layer Meteorol., 114(2), 245-285.

Lopez, F., and Garcia, M.H. (2001). "Mean flow and turbulence structure of open-channel flow through emergent vegetation.” J. Hydraul. Eng., 127(5), 392-402.

Nepf, H. (2012). “Hydrodynamics of vegetated channels.” J. Hydraul. Res., 50(3), 262-279.

Nikora, V.I., Goring, D.G., McEwan, I., and Griffiths, G. (2001). "Spatially-averaged open-channel flow over a rough bed.” J. Hydraul. Eng., 127(2), 123-133.

Nikora, V.I., McEwan, I.K., McLean, S.R., Coleman, S.E., Pokrajac, D., and Walters, R. (2007a). "Double-averaging concept for rough-bed open-channel and overland flows: Theoretical background.” J. Hydraul. Eng., 133(8), 873-883.

Nikora, V., McLean, S., Coleman, S., Pokrajac, D., McEwan, I., Campbell, L., Aberle, J., Clunie, D., and Koll, K. (2007b). "Double-averaging concept for rough-bed open-channel and overland flows: applications.” J. Hydraul. Eng., 133(8), 884-895.

Nikora, V., and Rowinski, P. (eds.) (2008). Rough-bed flows in geophysical, environmental, and engineering systems: Double-Averaging Approach and its applications. Special Issue. Acta Geophysica, 56(3), 529-934.

Papanicolaou, A.N., Dermisis, D., and Elhakeem, M. (2011). "Investigating the Role of Clasts on the Movement of Sand over Gravel Bed Rivers.” J. Hydraul. Eng., 137(9), 871-883.

Pedras, M.H.J., and de Lemos, M.J.S. (2000). "On the definition of turbulent kinetic energy for flow in porous media.” Int. Comm. Heat and Mass Transfer, 27(2), 211-220.

Poggi, D., Katul, G.G. and Albertson, J.D. (2004). "Momentum transfer and turbulent kinetic energy budgets within a dense model canopy.” Boundary-Layer Meteorol., 111, 589-614.

Pokrajac, D., McEwan, I.K., and Nikora, V. (2008). "Spatially averaged turbulent stress and its partitioning.” Exp. Fluids, DOI: 10.1007/s00348-008-0463.

Radice, A., Ballio, F. 2008. Double-average characteristics of sediment motion in one-dimensional bed load. Acta Geophysica, 56(3), 654-668, doi:10.2478/s11600-008-0015-0.

Raupach, M.R., and Shaw, R.H. (1982). "Averaging procedures for flow within vegetation canopies.” Boundary-Layer Meteorol., 22, 79-90.

Torquato, S. 2002. Random Heterogeneous Materials: Microstructure and Macroscopic Properties. Springer-Verlag, New York.

Wilson, N.R., and Shaw, R.H. (1977). “A higher order closure model for canopy flow.” J. Appl. Meteorology, 16, 1197-1205. 
606

607

608

609

610

611

612

613

614

615

616

617

618

619

620

621

622

623

624

625

626

627

628

629

630

631

632

633

634

635
Figure captions for the paper

“Spatially-averaged flows over mobile rough beds: definitions, averaging theorems, and conservation equations"

\author{
by V. Nikora, F. Ballio, S. Coleman, D. Pokrajac
}

Fig. 1. A sketch showing a local co-ordinate system and a spatial averaging domain. Note that below the roughness tops the averaging domain includes both fluid and fixed or mobile bed surface.

Fig. 2. A sketch for porosities for mobile-bed conditions (sediment transport and a moving plant), showing an averaging domain and embedded mobile and fixed objects (a), time evolution of solid object positions within the averaging domain: an example for the $x$-axis (b), spatial porosity changing in time (c), and time porosity changing along the flow (d). Black colour defines a particle that does not move within $T_{o}$ (i.e., a 'solid island' within the spatial averaging domain $V_{o}$ ); grey colour defines mobile and fixed particles that do not cross the averaging domain; and patterned objects define mobile particles and a waving plant that move through the averaging domain. In this example $\phi_{T m} \equiv 1$ (i.e., a part of $V_{o}$ is occupied by fluid at any time), while $\phi_{V m}<1$ (the black particle represents a solid island within $V_{o}$ ). 
636

637

638

639

640

641

642

643

644

645

646

647

648

649

650

651

652

653

654

655

656

657

658

659

660

661

662

663

664

665

666

667

668

669

670

671

672

673

674

675

676

677

678

679

680

681

682

683

684

685

686

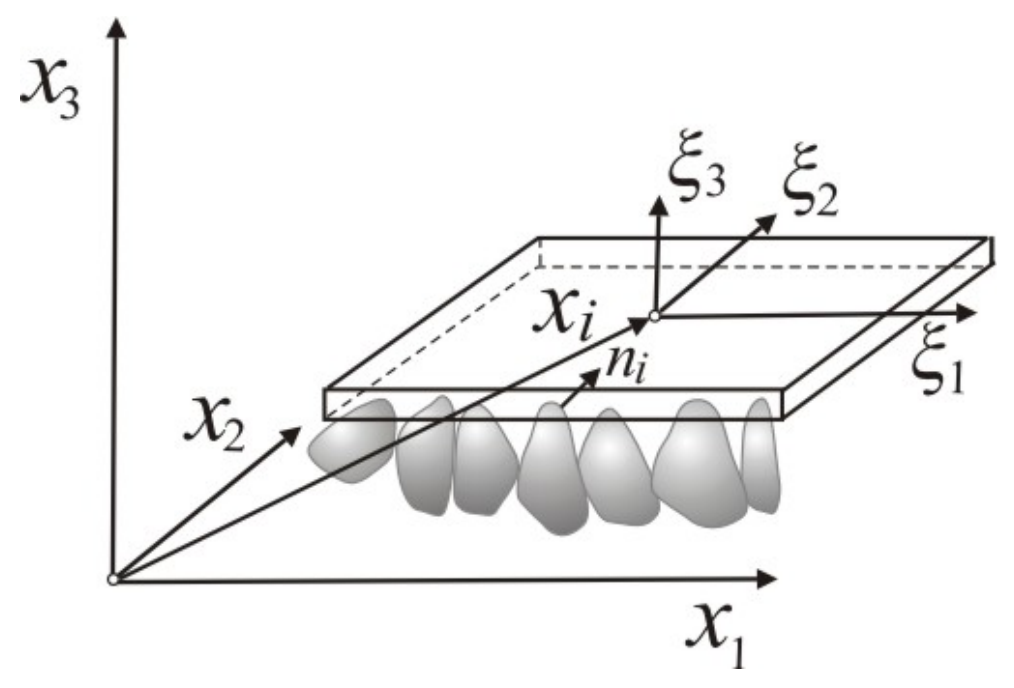

Fig. 1. A sketch showing a local co-ordinate system and a spatial averaging domain. Note that below the roughness tops the averaging domain includes both fluid and fixed or mobile bed surface. 
(a)

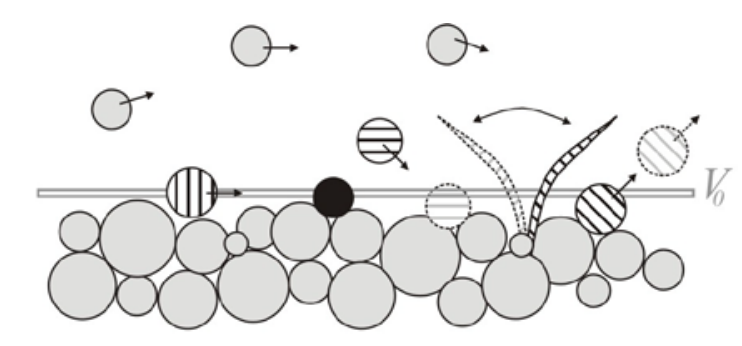

(b)

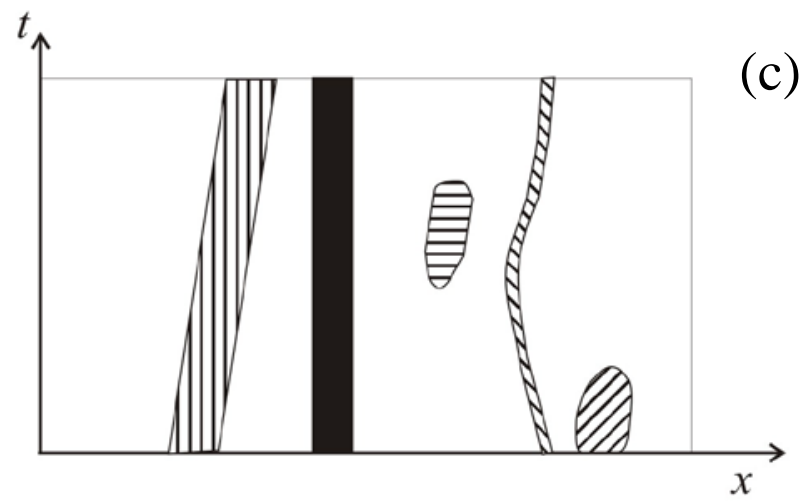

(d)

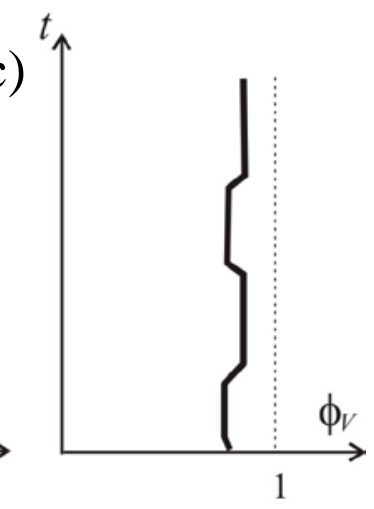

Fig. 2. A sketch for porosities for mobile-bed conditions (sediment transport and a moving plant), object positions within the averaging domain: an example for the $x$-axis (b), spatial porosity changing in time (c), and time porosity changing along the flow (d). Black colour defines a particle that does not move within $T_{o}$ (i.e., a 'solid island' within the spatial averaging domain $V_{o}$ ); grey colour defines mobile and fixed particles that do not cross the averaging domain; and patterned objects define mobile particles and a waving plant that move through the averaging domain. In this example $\phi_{T m} \equiv 1$ (i.e., a part of $V_{o}$ is occupied by fluid at any time), while $\phi_{V m}<1$ (the black particle represents a solid island within $V_{o}$ ). 